\title{
A pesquisa sobre Alfred North Whitehead no Brasil
}

Lília Dias Marianno ${ }^{1}$

\section{RESUMO:}

Este artigo pretende apresentar, em formato de antologia de textos, um mapeamento cronológico da produção acadêmica em teses e dissertações sobre Whitehead e em diálogo com o Pensamento Processual realizada por brasileiros, com ênfase na produção em língua portuguesa a partir dos anos 80 . Divide-se em teses e dissertações focadas em Whitehead e Pensamento Processual, teses e dissertações que dialogam com Whitehead e Pensamento Processual e pesquisas em andamento. Foram coletados dados por meio das seguintes plataformas: Plataforma Lattes do CNPQ, Plataforma Sucupira, Catálogo de Teses e Dissertações da CAPES, Biblioteca Digital Brasileira de Teses e Dissertações (BDTD), Grupos de Trabalho da ANPOF, além dos bancos de teses e dissertações de algumas universidades específicas. Os trabalhos elencados restringem-se à produção Brasileira (dentro e fora do país) e no Brasil (mesmo quando realizada por estrangeiros) e emitem uma fotografia datada até setembro de 2019. As descrições das pesquisas utilizam extratos de textos dos resumos das mesmas, compilando os textos dos próprios autores.

PALAVRAS-CHAVE: Alfred North Whitehead; Pensamento Processual no Brasil; Mapeamento de Pesquisa Acadêmica.

\begin{abstract}
This article intends to present, in the form of anthology of texts, a chronological mapping of academic production in theses and dissertations on Whitehead and in dialogue with the Processual Thought carried out by Brazilians, with an emphasis on the production in Portuguese language from the years 80 . It is divided into theses and dissertations focused on Whitehead and Processual Thinking, theses and dissertations that dialogue with Whitehead and Processual Thinking and research in progress. Data were collected through the following platforms: Platforma Lattes from CNPQ, Plataforma Sucupira, CAPES Theses and Dissertations Catalog, Brazilian Digital Library of Theses and Dissertations (BDTD), ANPOF Working Groups, in addition to thesis and dissertation banks of some specific universities. The listed works are restricted to Brazilian production (inside and outside the country) and in Brazil (even when performed by foreigners) and issue a photograph dated until September 2019. The research descriptions use extracts from texts from their abstracts , compiling the texts of the authors themselves.
\end{abstract}

KEYWORDS: Alfred North Whitehead; Brazilian Processual Thought; Academic Research Mapping.

\section{INTRODUÇÃO}

Antes de iniciarmos esta pesquisa, cuidamos para não confundir o Whitehead de nosso objetivo com outros homônimos que existem na literatura inglesa, na sociologia política e também na matemática e da álgebra, como é o caso de John Whitehead.

\footnotetext{
${ }^{1}$ Doutoranda do Programa de Pós-Graduação em História das Ciências e das Técnicas e Epistemologia da Universidade Federal do Rio de Janeiro (CCMN-NCE-HCTE).
} 
O acesso aos textos das teses e dissertações nem sempre nos atendeu como precisávamos, já que várias pesquisas são mais antigas que a exigência dos acervos digitais nas universidades e só possuem exemplares físicos nos respectivos acervos.

\section{1) PESQUISAS SOBRE ALFRED NORTH WHITEHEAD}

\section{1) 1985 - Filosofia [Tese]}

MIKOWSKI, Tadeu Cristovam. A religião segundo Alfred North Whitehead. Programa de Pós-Graduação em Filosofia Contemporânea. Pontificia Studiorum Universitas As Thoma Aq in Urbe. Roma.

Localização: não encontrada

Descrição: não encontrada

Palavras-chave: não encontrada

\section{2) 2004 - Psicologia [Dissertação]}

BATISTA, Jucenei. O fluxo da experiência em Whitehead. Programa de Pós-Graduação em Psicologia. Universidade Federal Fluminense (UFF). Niterói.

Localização: Biblioteca central do campus Gragoatá - UFF

Descrição: não encontrada

Palavras-chave: não encontrada

\section{3) 2011 - Filosofia [Dissertação]}

SILVA, Ednaldo Isidoro da. A cosmologia especulativa de Alfred North Withehead: sobre os princípios, as causas, a hierarquia e a ordem do universo orgânico. Programa de Pós-Graduação em Filosofia. Universidade Federal de Pernambuco (UFPE). Recife.

Localização: Biblioteca Central da UFPE

Descrição: não encontrada

Palavras-chave: não encontrada

\section{4) 2013 - Filosofia [Tese]}

RIBEIRO, Fernando Maia Freire. Individuação e inocência: composições com Simondon e Whitehead. Programa de Pós-Graduação em Filosofia. Universidade do Estado do Rio de Janeiro (UERJ). Rio de Janeiro. 
Localização: Biblioteca Digital de Teses e Dissertações da UERJ

Descrição: A pesquisa analisa a individuação apontando outra concepção de indivíduo, fora do dualismo antagonista. O pensamento especulativo de Whitehead e sua insistência pelo processo seria um antídoto à filosofia da individuação?

Palavras-chave: Modos. Híbridos. Inocência. Individuação. Whitehead. Simondon.

\section{5) 2013 - Epistemologia [Tese]}

GIUSTI, Marcos Vinício Guimarães. Da substância ao processo: a mudança da base metafísica da ciência no século XX. Programa de Pós-Graduação em História das Ciências e das Técnicas e Epistemologia da Universidade Federal do Rio de Janeiro (UFRJ). Rio de Janeiro.

Localização: Biblioteca Central CCMN/UFRJ - HCTE

Descrição: Apresenta a necessidade de uma mudança na base metafísica da ciência contemporânea. Argumenta-se que a Filosofia do Processo oferece os melhores conceitos metafísicos para se elaborar uma ontologia da mecânica quântica.

Palavras-chave: Ciência. Mecânica Quântica. Filosofia do processo. Heisenberg. Whitehead

\section{6) 2013 - Teologia [Dissertação]}

DANILAS, Sergio. O Deus que se relaciona: em uma perspectiva do processo. Programa de Pós-Graduação em Teologia. Pontifícia Universidade Católica do Paraná (PUC-PR). Curitiba.

Localização: Biblioteca Central da PUC-PR

Descrição: A pesquisa propõe o uso da filosofia do processo para estabelecer diálogo e relações entre a teologia cristã e as ciências da natureza e refletir sobre a magnitude das relações entre Deus e o mundo.

Palavras-chave: Religião e ciência. Teologia e ciências naturais. Whitehead. Processo. Deus e o mundo. Kenosis.

\section{7) 2018 - Administração [Tese]}

CHAERKI, Karine Francisconi. As organizações como eventos: apreensão da dinâmica do movimento organizacional em sua dimensão temporal. Programa de PósGraduação em Administração. Universidade Federal do Paraná (UFPR). Curitiba. Localização: Biblioteca Central da UFPR 
Descrição: Apresenta a Filosofia do Processo aplicada aos estudos organizacionais, procurando mostrar a organização como evento e como processo contínuo de transformação. Palavras-Chave: Processos organizacionais. Dimensões. Eventos, Whitehead. Filosofia do Processo.

\section{2) PESQUISAS EM DIÁlOGO COM WHITEHEAD}

\section{8) 1992 - Economia [Dissertação]}

FUKS, Mauricio. Considerações preliminares sobre a introdução do conceito de entropia na ciência econômica. Programa de Pós-Graduação em Economia. Fundação Getúlio Vargas (FGV). Rio de Janeiro.

Localização: Biblioteca da FGV-EPBE

Descrição: A pesquisa apresenta a noção de entropia em diálogo com teóricos como Robert M. Solow, Keneth E. Boulding, Herman Daly e trabalha em diálogo com temas whiteheadianos como: o concreto mal colocado, leis da termodinâmica, abstração, espaço e tempo.

Palavras-chave: Economia. Entropia. Teoria econômica. Evolução.

\section{9) 1993 - Educação [Dissertação]}

NETO, Pedro Antonio de Lima. A ideia de universidade numa universidade que nasce. Programa de pós-graduação em Educação da Universidade Federal de Santa Catarina (UFSC). Florianópolis.

Localização: Repositório Institucional da UFSC

Descrição: Investiga o conceito básico de universidade, analisando quatro modelos de universidades modernas do mundo ocidental: a alemã de Humboldt, a inglesa de Newman, a americana de Whitehead, a francesa de Napoleão.

Palavras-chave: Universidade. Educação. Interdisciplinaridade.

\section{0) 2001 - Matemática [Tese]}

ROQUE, Tatiana Marins. Ensaio sobre a gênese das ideias matemáticas. Programa de pós-graduação em Engenharia de Produção. Universidade Federal do Rio de Janeiro. (UFRJ). Rio de Janeiro.

Localização: Biblioteca do Centro Tecnológico da Escola Politécnica UFRJ Descrição: não encontrada 
Palavras-chave: Filosofia da matemática. História da matemática. Sistemas dinâmicos.

\section{1) 2005 - Psicologia [Tese]}

IZHAKI, Fania Goltsman. Transformações de si: uma leitura dos escritos de Winnicott priorizando processos e relações. Programa de Pós-Graduação em Saúde Coletiva da Universidade do Estado do Rio de Janeiro (UERJ).

Localização: Biblioteca Digital de Teses e Dissertações da UERJ

Descrição: A pesquisa realiza uma leitura dos escritos de Winnicott relacionados à filosofia processual de Whitehead, estabelecendo afinidades entre os dois autores com o objetivo de sistematizar conceitos, priorizando a abordagem relacional e processual em relação ao conceito de self.

Palavras-chave: Winnicott. Whitehead. Filosofia do processo. Transicionalidade. Apropriação.

\section{2) 2007 - Epistemologia [Dissertação]}

CARVALHO, Nelson Job Vasconcelos de. A diferença emaranhada: travessias entre a filosofia da diferença e a física da consciência. Programa de Pós-Graduação em História das Ciências e das Técnicas e Epistemologia da Universidade Federal do Rio de Janeiro (UFRJ).

Descrição: A partir da articulação entre os pensamentos de Deleuze, Heráclito, Spinoza, Leibniz, Nietzsche, Tarde, Bergson, Whitehead propõe novas abordagens para a clínica, a filosofia da ciência e para a teoria unificadora da física.

Localização: Biblioteca Central do Centro Tecnológico da Escola Politécnica da UFRJ Palavras-chave: Filosofia. Antropologia. Psicologia. Física. Cosmologia. Literatura.

\section{3) 2012 - Epistemologia [Tese]}

CARVALHO, Nelson Job Vasconcelos de. Rumo à Ontologia Onírica: confluências entre magia, filosofia e ciência. Programa de pós-graduação em História das Ciências e das Técnicas e Epistemologia. Rio de Janeiro. Universidade Federal do Rio de Janeiro, UFRJ.

Descrição. A pesquisa interpela o dualismo e a dicotomia entre os campos do saber, no próprio ser humano e nas suas manifestações. Propõe uma postura de coexistência 
integrada entre mundo e homem, de cosmos e consciência, do um e do múltiplo e também uma nova teoria dos sonhos.

Palavras-chave: Ciência. Filosofia. Hermetismo. Magia. Sonho. Transdisciplinaridade

\section{4) 2013 - História [Dissertação]}

BARBOSA, Mohana Ribeiro. Revolução científica e nascimento da ciência experimental em Alexandre Koyré. Programa de Pós-Graduação em História. Universidade Federal de Goiás (UFG). Goiânia.

Localização: Biblioteca Central da UFG

Descrição: A pesquisa busca compreender a definição de Koyré à ciência experimental do século XVII, explorando a importância deste conceito no interior da historiografia das ciências.

Palavras-chave: Historiografia das ciências. Filosofia das ciências. Positivismo.

Alexandre Koyré.

\section{5) 2014 - Filosofia [Dissertação]}

FREITAS, Jadson Alves de. Substâncias e relações em Leibniz: inspirações metafísicas para o pensamento filosófico nos séculos XX e XXI. Programa de Pós-Graduação em Filosofia. Universidade de Brasília (UnB).

Descrição: analisa as bases da monadologia de Leibniz como intuição para a formação de uma ideia geral sobre sistemas compostos por mônadas, contrastando com o pensamento sobre monadologia de Tarde, Whitehead, Latour, Schaffer e Quine.

Localização: Repositório Institucional da UnB

Palavras-chave: Mônada. Relação. Holismo. Compossibilidade. Metafísica.

\section{6) 2015 - Letras [Tese]}

RIBEIRO, Rodrigo Petronio. Mesons: Ontologia. Programa de Pós-Graduação em Teoria da Literatura e Literatura Comparada da Universidade Estadual do Rio de Janeiro (UERJ).

Descrição: A pesquisa se propõe a descrever e fundamentar a teoria da mesologia como uma teoria geral dos meios [mesons] e das mediações ou uma ontologia dos meios, fornecendo parâmetros conceituais para a compreensão de fenômenos heterogêneos, sejam eles de ordem cultural ou natural, física ou metafísica.

Palavras-chave: Mesons. Meios. Teoria Global dos Meios. Ontologia. Cosmologia. 


\section{7) 2015 - Filosofia [Tese]}

FIGUEIREDO, Fernando Padrão de. A superfície e a dobra: as condições do acontecimento em Gilles Deleuze. Programa de Pós Graduação em Filosofia da Universidade Federal do Rio de Janeiro (UFRJ).

Localização: Biblioteca depositária do IFCS - UFRJ

Descrição: Investiga a noção de acontecimento na filosofia de Gilles Deleuze, tendo como ponto de partida o pensamento de Bréhier sobre o estoicismo antigo, adentrando o pensamento de Whitehead para a construção da noção de conceito incorporal na obra $O$ que é $a$ filosofia?

Palavras-chave: Acontecimento. Incorporal. Gilles Deleuze. Maneirismo. Filosofia Contemporânea Francesa.

\section{8) 2016 - Estudos de CTS/Astronomia [Dissertação]}

COUTO, Walter Eler do. Preensão das estrelas: astronomia amadora, ciência conectada e Teoria Ator-Rede. Programa de Pós-Graduação em Cultura Contemporânea. Universidade Federal do Mato Grosso (UFMT). Cuiabá.

Localização: Biblioteca central da Universidade Federal do Mato Grosso

Descrição: Apresenta subversão proposta pelo sistema de Ciência Conectada no modo como se dá produção científica e a difusão de seus dados. Teoriza o debate entre as Epistemes Contemporâneas refletindo sobre o relacionamento político-epistemológico entre cientistas profissionais e amadores.

Palavras-Chave: Astronomia Amadora. Ciência Conectada. Colaboração. Comunicação Científica. Cibercultura.

\section{9) 2016 - Educação [Dissertação]}

LANZA, Maria Lucia. Sobre o bom professor: estudo de caso em uma escola da rede estadual paulista com alunos do ensino médio. Programa de Pós-graduação em Educação. Universidade Federal de São Paulo (USP).

Localização: Biblioteca Central da Universidade Federal de São Paulo. 
Descrição: Analisa a visão de alunos do ensino médio sobre seus bons professores e investiga a perspectiva dos estudantes sobre a atuação de professores que eles consideram competentes.

Palavras-Chave: Docência. Bom professor. Aluno do ensino médio. Rede estadual de ensino de São Paulo. Educação básica.

\section{0) 2016 - Direito [Dissertação]}

MACIEL, Otávio Souza e Rocha Dias. A systems theory reading of Kelsen: Global law and Politics beyond the Age of the Correlate. European Academy of Legal Theory. Frankfurt am Main, Goethe-Universität Frankfurt am Main. [Original não traduzido para o português]

Descrição: Partindo da análise de Meillassoux sobre a necessidade da contingência e da filosofia do processo de Whitehead, a pesquisa trabalha a teoria dos sistemas de Luhmann como uma poderosa aliada à teoria pura do direito de Kelsen para compreender o estado e o direito global.

Palavras-chave: Teoria do Direito. Metafísica da sociedade. Teoria Pura do Direito. Direito Global. Teoria de Sistemas.

\section{1) 2017 - Sociologia [Tese]}

\section{VARGAS, Felipe. "Você precisa estar na mata": entre saberes e modos de} engajamento nas práticas de conservação da biodiversidade no norte-amazônico brasileiro. Programa de Pós-Graduação em Sociologia. Universidade Federal do Rio Grande do Sul (UFRS). Porto Alegre.

Localização: Repositório digital LUME UFRS

Descrição: Apresenta o encontro de saberes entre biólogos, engenheiros florestais, ecólogos, ribeirinhos, indígenas e a mata no norte-amazônico brasileiro. Sociedade e ambiente se reconfiguram aprendendo novas maneiras de experienciar a realidade.

Palavras-chave: Conservação da biodiversidade. Amazônia. Sociologia do conhecimento. Desejo e crença.

\section{2) 2017 - Filosofia [Dissertação]}


SANTOS, Bernardo de Carvalho Tavares dos. Metafísica e exterioridade. Jean Wahl e o gosto empirista de Deleuze. Programa de Pós-Graduação em Filosofia. Universidade Brasília (UnB).

Localização: Repositório institucional da UNB

Descrição: Investiga as marcas fundamentais de um empirismo metafísico diretamente influenciado pelos pensadores Jean Wahl, Bergson, William James e Whitehead no pensamento de Deleuze.

Palavras-chave: Gilles Deleuze, 1925-1995; Empirismo; Metafísica; Filosofia - história 23) 2019 - Ciências da Religião [Tese]

BEZERRA, Karina Oliveira. Paganismo contemporâneo no Brasil : a magia da realidade. Programa de Pós-graduação em Ciências da Religião. Universidade Católica de Pernambuco (UNICAP). Recife.

Localização: Biblioteca digital de teses e dissertações da UNICAP

Descrição: O paganismo é um movimento religioso contemporâneo que acredita em vários deuses, sendo a natureza sacralizada e cultuada em ritos sazonais, com adaptações para a vida na sociedade moderna. A magia é o elo fundador e aglutinador do paganismo. É possível ver a magia como o "processo" de Whitehead.

Palavras-chave: Paganismo. Religiões - História. Realidade. Magia.

\section{3) PESQUISAS EM ANDAMENTO}

\section{i. Sobre Whitehead}

FERRARI, Francesco Maria. Towards a Metaphysics of Processes. [Pós-Doutorado] Programa de Pós-Graduação em História da Ciência, Lógica e Epistemologia da Universidade Estadual de Campinas (CLE/IFCH-UNICAMP).

FREITAS, Jadson Alves de Freitas. Whitehead e Simondon. [Doutorado] Programa de Pós-Graduação em Filosofia. Universidade de Brasília (PPGF-UnB). Orientador: Hilan Nissior Bensusan.

MARIANNO, Lília Dias. A ética relacional de Alfred North Whitehead na mediação de conflitos. [Doutorado] Programa de pós-graduação em história das ciências e das técnicas e epistemologia. Universidade Federal do Rio de Janeiro (HCTE-UFRJ). Orientadores: Mércio Pereira Gomes e Evandro Vieira Ouriques.

RIBEIRO, Rodrigo Petrônio. Alfred North Whitehead e as ontologias e cosmologias contemporâneas. [Pós-Doutorado] Programa de Pós-Graduação em Tecnologias da 
Inteligência e Design Digital. Pontifícia Universidade Católica de São Paulo (TIDDPUCSP).

SILVA E SILVA, Fernando. O fim da natureza: Alfred North Whitehead e Isabelle Stengers. [Doutorado] Programa de Pós-Graduação em Filsofia. Pontifícia Universidade Católica do Rio Grande do Sul (PUCRS). Orientador: Norman Roland Madarasz.

\section{ii. Em diálogo com Whitehed}

ARNAUT, André Roberto Tonussi. Ontologia plana. [Tese]; BARROS, Bárbara de. Whitehead, Stengers e Bataille. [Tese]; MACIEL, Otávio S. R. D. Tratado de Metametafísica: Teoria e Constituição [Tese]; GABRIEL, Alice de Barros. Feminismos materiais [Tese]; OSMAN, Zahra. Derrida e pensamento pós-colonial. [Tese]

Todas estas pesquisas estão em desenvolvimento no Programa de Pós-Graduação em Filosofia na Universidade de Brasília sob orientação do professor Dr. Hilan Nissior Bensusan.

\section{4) RESUMO}

\section{TABELA 1.}

Pesquisa whiteheadiana produzida no Brasil e no exterior e produzida por brasileiros (ou estrangeiros) estudando no Brasil

\begin{tabular}{|l|l|l|}
\hline Sobre Whitehead & & \% \\
\hline Teses & 4 & 57,1 \\
\hline Dissertações & 3 & 42,9 \\
\hline TOTAL & $\mathbf{7}$ & $\mathbf{1 0 0}$ \\
\hline
\end{tabular}

\begin{tabular}{|l|l|l|}
\hline Em diálogo com Whitehead & & $\%$ \\
\hline Teses & 7 & 43,75 \\
\hline Dissertações. & 9 & 56,25 \\
\hline TOTAL & $\mathbf{1 6}$ & $\mathbf{1 0 0}$ \\
\hline TOTAL DE PESQUISAS & $\mathbf{2 3}$ & \\
\hline
\end{tabular}

\begin{tabular}{|l|l|l|}
\hline Pesquisas em andamento* & & $\%$ \\
\hline Sobre Whitehead & 5 & $50 \%$ \\
\hline Em diálogo com Whitehead & 5 & $50 \%$ \\
\hline TOTAL EM ANDAMENTO (estimado) & $\mathbf{1 0}$ & \\
\hline
\end{tabular}

TABELA 2. 


\section{Localização das pesquisas}

\begin{tabular}{|l|l|l|l|l|l|l|}
\hline & Universidades & SIGLAS & ESTADO & QTD & $\begin{array}{l}\text { ESTA- } \\
\text { DOS }\end{array}$ & REGIÕES \\
\hline 1 & Universidade São Tomás de Aquino (Roma) & PUCSTA & Ext & 1 & 1 & \\
\hline 2 & Universidade Federal Fluminense & UFF & RJ & 1 & & SD \\
\hline 3 & Universidade do Estado do Rio de Janeiro & UERJ & RJ & 3 & & SD \\
\hline 4 & Universidade Federal do Rio de Janeiro & UFRJ & RJ & 5 & & SD \\
\hline 5 & Fundação Getúlio Vargas - RJ & FGV & RJ & 1 & & SD \\
\hline 6 & Universidade Federal de São Paulo & UNIFESP & SP & 1 & 10 & SD \\
\hline 7 & Universidade Federal de Santa Catarina & UFSC & SC & 1 & & SUL \\
\hline 8 & Universidade Federal do Rio Grande do Sul & UFRS & RS & 1 & & SUL \\
\hline 9 & Pontifícia Universidade Católica Paraná & PUC-PR & PR & 1 & & SUL \\
\hline 10 & Universidade Federal do Paraná & UFPR & PR & 1 & 4 & SUL \\
\hline 11 & Universidade Federal de Goiás & UFG & GO & 1 & & CO \\
\hline 12 & Universidade de Brasília & UNB & DF & 2 & & CO \\
\hline 13 & Universidade Federal do Mato Grosso & UFMT & MT & 1 & 4 & CO \\
\hline 14 & Universidade Católica de Pernambuco & UNICAP & PE & 1 & & NE \\
\hline 15 & Universidade Federal de Pernambuco & UFP & PE & 1 & 2 & NE \\
\hline 16 & Goethe-Universität Frankfurt am Main. & GUFM & Ext & 1 & 1 & \\
\hline & TOTAL DE PESQUISAS & & 9 estados & $\mathbf{2 3}$ & & \\
\hline
\end{tabular}

TABELA 3.

Interdisciplinaridade do diálogo com Whitehead

\begin{tabular}{|l|l|l|}
\hline & Interdisciplinaridade & QTD \\
\hline 1 & Administração & 1 \\
\hline 2 & Astronomia (Estudos CTS) & 1 \\
\hline 3 & Ciências da Religião & 1 \\
\hline 4 & Direito & 1 \\
\hline 5 & Economia & 1 \\
\hline 6 & Educação & 2 \\
\hline 7 & Epistemologia & 3 \\
\hline 8 & Filosofia & 6 \\
\hline 9 & História & 1 \\
\hline 10 & Letras & 1 \\
\hline 11 & Matemática & 1 \\
\hline 12 & Psicologia & 2 \\
\hline 13 & Sociologia & 1 \\
\hline 14 & Teologia & 1 \\
\hline & TOTAL DE PESQUISAS & $\mathbf{2 3}$ \\
\hline
\end{tabular}

\section{CONCLUSÃO}


Em tempos de comunicação digital algumas considerações são oportunas sobre a visibilização da pesquisa sobre Whitehead produzida no Brasil ou por brasileiros, lembrando que não tivemos intenção de elencar a produção bibliográfica em livros e artigos. 1) Whitehead não é um autor popularizado no Brasil, a publicação de apenas três de seus títulos publicados no Brasil acontece com intervalo de décadas entre uma e outra. Há outras obras em português publicadas em Portugal, mas o acesso do pesquisador brasileiro de nível de graduação ainda não é pleno a estas obras.

2) A difusão de Whitehead no Brasil se dá sensivelmente por seus discípulos mais conhecidos no Brasil como Gilles Deleuze e Bruno Latour.

3) A pesquisa brasileira sobre Whitehead e em diálogo com Whitehead existe, é original e criativa, dialógica, interdisciplinar e transdisciplinar, embora permaneça praticamente desconhecida.

4) Em 2011 quando iniciamos este escaneamento, era praticamente impossível encontrar estas referências. Continua sendo difícil rastreá-las, mas constatamos que a quantidade de pesquisas em andamento atualmente chega a quase $50 \%$ de tudo que foi produzido sobre este autor de 1985 até 2019.

5) Apesar de a bibliografia completa de Whitehead estar disponível em inglês pelo Internet Archive Search (Archive.org), com a ajuda do Centro de Filosofia da Universidade de Lisboa conseguimos a doação de uma quantidade considerável de Processo e Realidade para serem doadas a bibliotecas de importantes centros de pesquisa pelo Brasil (IFCS-UFRJ; CCMN-UFRJ, IM-UFRJ; CBPF; UEFS; BN; entre outras.)

6) A partir da 12th International Whitehead Conference ocorrida em 2019 na UnB os pesquisadores brasileiros aqui mencionados, em substancial representatividade, começam a se articular num network orgânico e processual em termos de relações de modo que futuros eventos para difusão da pesquisa whiteheadiana sejam realizados em todo o país.

7) Há uma lacuna de produção acadêmica sobre Whitehead adotando a abordagem da sustentabilidade, o que se faz urgente pelos episódios que temos testemunhados sobre as condições climáticas e desflorestamento.

8) O presente mapeamento não é exaustivo. Há certamente muitas outras pesquisas em níveis de teses e dissertações envolvendo Whitehead que não foi possível rastrear nas bases digitais e que podem compor uma versão mais ampla deste trabalho. Solicitamos 
que outros pesquisadores sobre Whitehead não mencionados aqui façam contato conosco para tal interação.

9) Queremos destacar a atuação do prof. Dr. Hilan Bensusan na orientação de grande parte dos atuais pesquisadores de mestrado e doutorado sobre Whitehead, da Pós-Graduação em Filosofia da Universidade de Brasília e na produção de livros sobre o assunto em co-autoria com seus estudantes, devidamente referenciados na bibliografia deste texto. O prof. Hilan é atualmente o catedrático mais engajado no Brasil com os estudos whiteheadianos.

Queremos agradecer ao Dr. Rodrigo Petrônio Ribeiro, Ddos Fernando Silva e Silva e ao Ddo. Otávio Maciel pelos importantes adendos a este texto, possibilitando maior justiça na visibilidade dos autores mencionados.

\section{REFERÊNCIAS BIBLIOGRÁFICAS}

(i) Bases de dados:

\section{ARCHIVE.ORG}

https://archive.org/search.php?query=Alfred\%20north\%20whitehead.

BIBLIOTECA DIGITAL DE TESES E DISSERTAÇÕES:

http://bdtd.ibict.br/vufind/

CATÁLOGO DE TESES E DISSERTAÇÕES CAPES MEC http://sdi.capes.gov.br/banco-de-teses/01_bt_index.html

GT ONTOLOGIAS CONTEMPORÂNEAS - ASSOCIAÇÃO NACIONAL DE PÓSGRADUAÇÃO EM FILOSOFIA - ANPOF http://anpof.org/portal/index.php/pt-BR/

INSTITUTO BRASILEIRO DE INFORMAÇÃO EM CIÊNCIA E TECNOLOGIA http://ibict.br

PLATAFORMA LATTES - CNPQ http://lattes.cnpq.br/

PLATAFORMA SUCUPIRA https://sucupira.capes.gov.br/sucupira/public/consultas/coleta

\section{(ii) Livros}


BENSUSAN, Hilan N.; FREITAS, Jadson Alves de. A diáspora da agência - ensaio sobre o horizonte das monadologias. Salvador: EDUFBA, 2018.

BENSUSAN, Hilan. Being Up for Grabs: On Speculative Anarcheology. London: Open Humanities Press, 2018.

\section{(iii) Teses e Dissertações}

BARBOSA, Mohana Ribeiro. Revolução científica e nascimento da ciência experimental em Alexandre Koyré. [Dissertação]. Programa de Pós-Graduação em História. Universidade Federal de Goiás. Goiânia, 2013, 110p. Disponível em: $<$ http://repositorio.bc.ufg.br/tede/bitstream/tde/3031/5/Dissertacao\%20Mohana\%20R\%20Barbosa.pdf> Acessado em: 17/08/2019.

BATISTA, Jucenei. O fluxo da experiência em Whitehead.[Dissertação]. Programa de pós-graduação em psicologia. Universidade Federal Fluminense. Niterói, 2004, 78p.

BEZERRA, Karina Oliveira. Paganismo contemporâneo no Brasil : a magia da realidade. [Tese] Programa de Pós-graduação em ciências da religião. Universidade Católica de Pernambuco. Recife, 2019, 498 p. Disponível em: <http://tede2.unicap.br:8080/handle/tede/1124> Acessado em: 26/09/2019.

CARVALHO, Nelson Job Vasconcelos de. A diferença emaranhada: travessias entre a filosofia da diferença e a física da consciência. [Dissertação] Programa de pósgraduação em história das ciências e das técnicas e epistemologia. Universidade Federal do Rio de Janeiro. Rio de Janeiro, 2007, 108p.

CARVALHO, Nelson Job Vasconcelos de. Rumo à Ontologia Onírica: confluências entre magia, filosofia e ciência. [Tese] Programa de pós-graduação em História das Ciências e das Técnicas e Epistemologia. Universidade Federal do Rio de Janeiro. Rio de Janeiro, 2012, 165p.

CHAERKI, Karine Francisconi. As organizações como eventos: apreensão da dinâmica do movimento organizacional em sua dimensão temporal. [Tese] Programa de Pós-Graduação em Administração. Universidade Federal do Paraná. Curitiba, 2018, 212p. Disponível em: <https://sucupira.capes.gov.br/sucupira/public/consultas/coleta/trabalhoConclusao/viewTrabalhoConclusao.jsf?popup=true\&id_trabalho $=5844981>$ Acessado em: 17/08/2019.

COUTO, Walter Eler do. Preensão das estrelas: astronomia amadora, ciência conectada e Teoria Ator-Rede. [Dissertação] Programa de Pós-Graduação em Cultura Contemporânea. Universidade Federal de Mato Grosso. Cuiabá, 2016, 260p. Disponível em:

$<$ https://www.academia.edu/26403187/Preens\%C3\%A3o_das_Estrelas_astronomia_amadora_ci\%C3\%AAncia_conectada_e_teoria_ator-rede $>$ Acessado em 26/09/2019. 
DANILAS, Sergio. O Deus que se relaciona: em uma perspectiva do processo. [Dissertação] Programa de Pós-Graduação em Teologia. Pontifícia Universidade Católica do Paraná. Curitiba, 2013, 135p.

FIGUEIREDO, Fernando Padrão de. A superfície e a dobra: as condições do acontecimento em Giles Deleuze. [Tese] Programa de Pós Graduação em Filosofia da Universidade Federal do Rio de Janeiro. Rio de Janeiro, 2015, 268 p. Disponível em: $<$ https://sucupira.capes.gov.br/sucupira/public/consultas/coleta/trabalhoConclusao/viewTrabalhoConclusao.jsf?popup $=$ true\&id_trabalho $=3520264>$ Acessado em: $17 / 08 / 2019$

FREITAS, Jadson Alves de. Substâncias e relações em Leibniz: inspirações metafísicas para o pensamento filosófico nos séculos XX e XXI. [Dissertação] Programa de Pós-Graduação em Filosofia. Universidade de Brasília. Brasíllia, 2014, 147p. Disponível em: <http://repositorio.unb.br/handle/10482/17452>. Acessado em 17/08/2019.

FUKS, Mauricio. Considerações preliminares sobre a introdução do conceito de entropia na ciência econômica. [Dissertação] Programa de Pós-Graduação em Economia. Fundação Getúlio Vargas. Rio de Janeiro, 1992, 91p. Disponível em: $<$ http:/hdl.handle.net/10438/133>. Acessado em: 20/08/2019.

GIUSTI, Marcos Vinício Guimarães. Da substância ao processo: a mudança da base metafísica da ciência no século XX. [Tese] Programa de Pós-Graduação em História das Ciências e das Técnicas e Epistemologia. Universidade Federal do Rio de Janeiro. Rio de Janeiro, 2013, 131p. Disponível em: <http://objdig.ufrj.br/10/teses/798964.pdf> Acessado em 17/08/2019.

IZHAKI, Fania Goltsman. Transformações de si: uma leitura dos escritos de Winnicott priorizando processos e relações. [Tese doutoral]. Programa de Pós-Graduação em Saúde Coletiva. Universidade do Estado do Rio de Janeiro. Rio de Janeiro, 2005, 193p. Disponível em: <http://www.bdtd.uerj.br/tde_busca/arquivo.php?codArquivo=7650 $>$. Acessado em: 16/08/2019.

LANZA, Maria Lucia. Sobre o bom professor: estudo de caso em uma escola da rede estadual paulista com alunos do ensino médio. [Dissertação] Programa de Pós-Graduação em Educação. Universidade Federal de São Paulo. São Paulo, 2016, 185p. Disponível em: <https://sucupira.capes.gov.br/sucupira/public/consultas/coleta/trabalhoConclusao/viewTrabalhoConclusao.jsf?popup=true\&id_trabalho=4441741\#>. Acessado em 19/08/2019.

MIKOWSKI, Tadeu Cristovam. A religião segundo Alfred North Whitehead. [Tese doutoral] Programa de Pós-Graduação em Filosofia Contemporânea. Pontificia Studiorum Universitas As Thoma Aq in Urbe. Roma, 1985.

NETO, Pedro Antônio de Lima. A ideia de universidade numa universidade que nasce. [Dissertação] Programa de Pós-Graduação em Educação da Universidade Federal de Santa Catarina. Florianópolis, 1993, 57p. Disponível em: 
$<$ https://repositorio.ufsc.br/bitstream/handle/123456789/111432/91024.pdf?sequence $=1 \&$ isAllowed $=\mathrm{y}>$. Acessado em: 19/08/2019.

RIBEIRO, Fernando Maia Freire. Individuação e inocência: composições com Simondon e Whitehead. [Tese] Programa de Pós-Graduação em Filosofia. Universidade do Estado do Rio de Janeiro. Rio de Janeiro, 2013, 224p. Disponível em: $<$ http://www.bdtd.uerj.br/tde_busca/arquivo.php?codArquivo=6562>. Acessado em: 20/08/2019.

RIBEIRO, Rodrigo Petrônio. Mesons: Ontologia. [Tese] Programa de Pós-Graduação em Teoria da Literatura e Literatura Comparada. Universidade Estadual do Rio de Janeiro [UERJ]. Rio de Janeiro, 2015, 497p. Disponível em: $<$ http://www.bdtd.uerj.br/tde_busca/arquivo.php?codArquivo=9666 $>$ Acessado em 26/09/2019.

ROQUE, Tatiana Marins. Ensaio sobre a gênese das ideias matemáticas. [Tese] Programa de Pós-Graduação em Engenharia de Produção. Universidade Federal do Rio de Janeiro. Rio de Janeiro, 2001, 263p.

SILVA, Ednaldo Isidoro da. A cosmologia especulativa de Alfred North Withehead: sobre os princípios, as causas, a hierarquia e a ordem do universo orgânico. [Dissertação]. Programa de Pós-Graduação em Filosofia. Universidade Federal de Pernambuco (UFPE). Recife, 2011, 112 p.

SANTOS, Bernardo de Carvalho Tavares dos. Metafísica e exterioridade. Jean Wahl e o gosto empirista de Deleuze. [Dissertação] Programa de Pós-Graduação em Filosofia. Universidade de Brasília. Brasília, 2017, 120p. Disponível em: $<$ http://repositorio.unb.br/handle/10482/23981> Acessado em: 16/08/2019.

VARGAS, Felipe. "Você precisa estar na mata": entre saberes e modos de engajamento nas práticas de conservação da biodiversidade no norte-amazônico brasileiro. [Tese] Programa de Pós-Graduação em Sociologia. Universidade Federal do Rio Grande do Sul. Porto Alegre, 2017, 219 p. Disponível em: <http://hdl.handle.net/10183/180598> Acessado em: 19/08/2019.

Recebido em: 05/10/2019

Aprovado em: 09/12/2019

* Esse trabalho é licenciado pela Creative Commons Attribution-NonCommercial 4.0 International License 